\title{
Contrast-induced acute kidney injury in patients undergoing cardiac resynchronization therapy-incidence and prognostic importance. Sub-analysis of data from randomized TRUST CRT trial
}

\author{
Jacek Kowalczyk • Radoslaw Lenarczyk • Oskar Kowalski • Tomasz Podolecki • \\ Pawel Francuz • Patrycja Pruszkowska-Skrzep • Mariola Szulik • Michal Mazurek • \\ Ewa Jedrzejczyk-Patej • Beata Sredniawa • Zbigniew Kalarus • \\ for the Triple-Site Versus Standard Cardiac Resynchronization Trial (TRUST CRT) Investigators
}

Received: 5 December 2013 / Accepted: 12 February 2014 / Published online: 14 March 2014

(C) The Author(s) 2014. This article is published with open access at Springerlink.com

\begin{abstract}
Introduction Because data on contrast-induced acute kidney injury (CI-AKI) in patients undergoing cardiac resynchronization therapy (CRT-D) are scarce, we aimed to assess the incidence, natural course and prognostic importance of this syndrome in CRT recipients.

Methods Study population consisted of 100 consecutive patients enrolled into the Triple Site Versus Standard Cardiac Resynchronization (TRUST CRT) trial, who were treated with CRT-D. Two patients were excluded up to 3 months after randomization and not analysed further. CI-AKI was defined as a rise in serum creatinine of at least $26.5 \mu \mathrm{mol} / \mathrm{L}(0.3 \mathrm{mg} /$ dL) within $48 \mathrm{~h}$ after contrast exposure, or at least $50 \%$ increase from the baseline value during index hospital stay with CRT-D implantation according to KDIGO Clinical Practice Guideline for Acute Kidney Injury.

Results Among 98 subjects of TRUST CRT trial, 10 patients (10.2\%) developed CI-AKI after CRT-D implantation. In patients with glomerular filtration rate (GFR) $<60 \mathrm{~mL} / \mathrm{min} /$ $1.73 \mathrm{~m}^{2}$ on admission, the incidence of CI-AKI was almost twofold $(15.4 \%)$ higher than in subjects with GFR $\geq 60$ $(8.3 \%)$. CRT-D recipients with CI-AKI had significantly higher mortality rate $(50.0 \%)$ compared to those without CI-AKI (17.0\%) during 30 months of follow-up (logrank
\end{abstract}

J. Kowalczyk $(\bowtie) \cdot$ R. Lenarczyk $\cdot$ O. Kowalski $\cdot$ T. Podolecki

P. Francuz · P. Pruszkowska-Skrzep • M. Szulik • M. Mazurek •

E. Jedrzejczyk-Patej $\cdot$ B. Sredniawa $\cdot$ Z. Kalarus

Department of Cardiology, Congenital Heart Diseases and

Electrotherapy, Silesian Center for Heart Diseases, Medical

University of Silesia, ul. Curie-Sklodowskiej 9, Zabrze, Poland

e-mail: jacekmed@yahoo.com $p=0.012$ ). Multivariate Cox regression analysis showed CI-AKI as significant and independent risk factor for death in CRT-D recipients (hazard ratio 5.71; $95 \%$ CI 5.16-6.26; $p=0.001$ ).

Conclusions Contrast-induced acute kidney injury is a serious and frequent procedural complication of CRT-D implantation with a significant negative influence on long-term survival. The results suggest that clinical evaluation regarding renal function should be considered in CRT-D recipients, both before and after device implantation.

Keywords Cardiac resynchronization therapy $\cdot$ Chronic kidney disease $\cdot$ Contrast-induced acute kidney injury $\cdot$ Heart failure $\cdot$ Renal function

\section{Introduction}

Cardiac resynchronization therapy (CRT) has become the standard of care for patients with symptomatic heart failure (HF), significant electrical dyssynchrony and moderate to severe left ventricular systolic dysfunction. Due to rapid progress in technology and broadening indications, CRT is being employed more and more widely worldwide, with the estimated implantation rate reaching 140 per million European inhabitants in 2011 [1]. According to the current recommendations, implantation of biventricular pacemaker should be preceded by detailed intraoperative assessment of coronary sinus anatomy, to allow further optimal selection of the target vessel for left ventricular lead [2]. Although alternative imaging techniques may be employed as well, balloon-occlusive contrast angiography of the coronary sinus tributaries is 
currently the gold standard. However, the use of contrast media, even if non-ionic and low-osmotic, is inevitably associated with the risk of contrast-induced acute kidney injury (CI-AKI) - an iatrogenic syndrome with potentially serious consequences demonstrated in some groups of patients [3-6]. Moreover, heart failure is one of the most important risk factors of CI-AKI occurrence and therefore the evaluation of this procedural complication seems to be especially important in patients receiving CRT [5-8]. In fact, data limited to the incidence of CI-AKI in a population of CRT-D recipients has been so far reported in only two studies. However, according to the authors' best knowledge, there is a lack of data on CIAKI prognostic value in this set of patients $[8,9]$. Therefore, we aimed to assess the incidence, natural course and prognostic importance of this syndrome in CRT recipients.

\section{Methods}

\subsection{Patients}

Study population consisted of patients enrolled into the Triple Site Versus Standard Cardiac Resynchronization (TRUST CRT) trial. TRUST CRT was a single-center, single-blind, parallel, randomized, clinical trial to test the hypothesis that triple-site (double-left single-right) pacing with defibrillator is superior over conventional CRT with defibrillator [10, 11]. Eligibility criteria included heart failure in NYHA class III-IV despite optimal pharmacotherapy, left ventricular ejection fraction $\leq 35 \%$, QRS duration $\geq 120 \mathrm{~ms}$ and significant ( $\geq 40 \mathrm{~ms}$ ) intra- or interventricular mechanical dyssynchrony. The primary end-point of the trial was the 6 months combined response rate in both groups, the secondary end-point will assess time to the first major adverse cardiac event after 3 years [10].

Between 2008 and 2010, 100 consecutive patients who met the inclusion criteria were enrolled and randomized in a 1:1 fashion to triple-site or conventional CRT with defibrillator (CRT-D). All patients were implanted with resynchronization systems with implantable defibrillator-cardioverter (InSync Sentry Model 7298, Medtronic, Minneapolis, USA). Standard, commercially available leads were used to pace/sense the atrium, right ventricle and left ventricle. A bipolar Yconnector (Lead Adaptor 2827, Medtronic) was employed to connect in parallel two left ventricular leads in the triple-site group. Atrioventricular and interventricular delays were optimized between the first and the third postoperative day under the echocardiographic guidance. Two patients were excluded up to 3 months after randomization and data of these patients were not analysed further.

The study protocol and procedural outcomes have been published previously $[10,11]$. The investigation conforms with the principles outlined in the Declaration of Helsinki.
The protocol of the trial was approved by the locally appointed ethics committee. Written informed consent has been obtained from all study participants.

\subsection{Procedural details}

In order to analyse the potential association between postoperative renal function and characteristics of implantation procedure, data on procedural details were collected and analysed. These included procedure duration (defined as skin-to-skin time), fluoroscopy time and exposure, volume of contrast medium used and final location of left ventricular lead. Lead location was considered optimal, if its tip was in non-apical, lateral or postero-lateral segment of the left ventricle (as assessed in right- and left-lateral oblique fluoroscopic projection). Iso-osmolar, non-ionic contrast medium (Visipaque, GE Healthcare A.S., Norway) was used in all patients to perform angiography of the coronary veins. Intravenous hydration with $0.9 \%$ sodium chloride solution $(0.5 \mathrm{~mL} / \mathrm{kg}$ per hour) was implemented before contrast exposure and continued for 6-12 $\mathrm{h}$ in all patients with glomerular filtration rate (GFR) $<60$. Metformin and potentially nephrotoxic drugs, e.g. NSAIDs, aminoglycosides, were avoided or discontinued for at least $48 \mathrm{~h}$ after contrast administration.

\subsection{Follow-up and renal function assessment}

Patients were followed 1 week, 1 month, 3 months and 6 months after randomization and every 6 months thereafter. Apart from demographic, clinical data collection and echocardiographic measurements, serum creatinine levels were assessed at baseline and 6 months after randomization. In all patients, at least three blood samples were taken for serum creatinine measurements at different time points during baseline hospitalization: on admission, 1 day after implantation and at discharge. Additional, daily measurements were collected in cases of post-operative renal dysfunction, as needed. One blood sample was taken for serum creatinine 6 months after randomization. Serum creatinine levels were subsequently used to calculate GFR, according to the abbreviated Modification of Diet in Renal Disease Study Group Equation proposed by National Kidney Foundation [12]. Baseline chronic renal disease was defined as GFR $<60 \mathrm{~mL} / \mathrm{min} /$ $1.73 \mathrm{~m}^{2}$ on admission. CI-AKI was defined as a rise in serum creatinine of at least $26.5 \mu \mathrm{mol} / \mathrm{L}(0.3 \mathrm{mg} / \mathrm{dL})$ within $48 \mathrm{~h}$ after contrast exposure, or at least $50 \%$ increase from the baseline value during index hospital stay with CRT-D implantation according to KDIGO Clinical Practice Guideline for Acute Kidney Injury [5, 6]. Response to CRT was defined as a reduction of at least one NYHA class after 6 months of biventricular pacing. 


\subsection{Major adverse events}

Data on potential adverse events were collected throughout the entire follow-up during scheduled and unscheduled visits, via telephone calls, fax and other media from patients, relatives, witnesses, death certificates, hospital records, outpatient notes, letters, device memory and all other available sources. This data was subsequently classified by two independent members of Adverse Event Adjudication Board, blinded to patients' treatment arm. Major adverse cardiac event was considered a composite of hospitalization for heart failure, heart transplantation or all-cause death. Death certificates, hospital records and device memory data were all used to assess cardiac mortality either as sudden death or death from coronary artery disease (CAD), including myocardial infarction, congestive heart failure, valvular heart disease, cardiomyopathy, ventricular arrhythmia as well as other reasons of cardiac arrest. Remote outcome was defined as total mortality or any other endpoint occurrence within the whole 30-month observation period.

\subsection{Statistical analysis}

Continuous parameters were expressed as medians with minimal and maximal values; categorical variables were presented as numbers and percentages. Comparative analysis between groups was performed using Mann-Whitney $U$ test for continuous variables and Chi-square or Fisher's exact test, as appropriate, for dichotomous parameters. Logrank tests were used to compare Kaplan-Meier curves plotted for cumulative survival. Independent predictors of death were identified with multivariate Cox regression model and expressed as hazard ratio with $95 \%$ confidence interval. Regression models were developed after the inclusion of all parameters with univariate association with any-cause death and next backward stepwise variable selection method was performed. Multivariate logistic regression was used to identify independent predictors of CI-AKI. Generalized linear models for binomial data were constructed to test for interactions between treatment assignment (conventional vs. triple-site CRT) and prognostic effect of CI-AKI. All tests were double-sided. $p$ value $<0.05$ was considered statistically significant. All analyses were performed using the software package Statistica (version 6.1, StatSoft Inc., Tulsa, OK, USA).

\section{Results}

\subsection{Incidence of CI-AKI among CRT-D recipients}

Among 98 subjects of TRUST CRT Trial, 10 patients $(10.2 \%)$ developed CI-AKI after CRT-D implantation. Additional analysis revealed that in patients with decreased GFR
$<60$ on admission the incidence of CI-AKI was almost twofold (15.4\%) higher than in subjects with GFR $\geq 60$ (8.3\%).

\subsection{Baseline and clinical characteristics of the study groups}

Patients with CRT-D, who developed CI-AKI after implantation procedure, had very similar baseline and clinical characteristics when compared to those who did not develop CI-AKI (Table 1). In fact, the only significant baseline difference between these two groups was serum creatinine level on admission, which was higher in CI-AKI group. On the other hand, both groups did not differ significantly with respect to GFR on admission (Table 1). Considering data at discharge, the CI-AKI group had significantly lower GFR and higher prevalence of digoxin prescribed. All other baseline and inhospital parameters presented in the Table 1 were very similar with non-significant differences between analysed groups. Results of multivariate logistic regression analysis showed that the only independent predictor of CI-AKI after CRT implantation was a high serum creatinine concentration at baseline (Table 2).

\subsection{Natural course of CI-AKI}

The analysis of natural course of renal function within particular study groups revealed that serum creatinine and median GFR did not change significantly within 6 months after CRTD implantation (Fig. 1). The separate analysis of intergroup differences showed that, similarly to the relation at discharge, the median value of serum creatinine remained significantly higher in CI-AKI group after 6 months of follow-up. The median value of GFR after 6 months was lower in CI-AKI group with a trend towards a significant difference (Table 1).

\subsection{Prognostic value of CI-AKI}

CRT-D recipients with CI-AKI had significantly higher mortality rate $(50.0 \%)$ compared to those without CI-AKI (17.0\%) during 30 months of follow-up (Table 1). Cumulative surviving in analysed groups was presented with KaplanMeier curves and their comparison with logrank test revealed significant difference with $p=0.012$ (Fig. 2).

All the parameters with univariate association with death and potential impact on mortality were incorporated into multivariate Cox regression model. Selection of the risk factors for all-cause death adjustment is presented in footnotes of Table 3. Multivariate Cox regression analysis using backward stepwise variable selection method showed peak oxygen consumption, QRS complex width, mitral effective regurgitant orifice area and CI-AKI as significant and independent risk factors for death in CRT-D recipients (Table 3). Prognostic effect of CI-AKI on mortality ( $p$ for interactions $=0.26$ ), cardiac mortality $(p=0.09)$ and $\operatorname{MACE}(p=0.92)$ was 
Table 1 Clinical characteristics of the study population and comparative analysis among CRT-D recipients with respect to CI-AKI occurrence

\begin{tabular}{|c|c|c|c|c|}
\hline & $\begin{array}{l}\text { A } \\
\text { Overall population }(n=98)\end{array}$ & $\begin{array}{l}\text { B } \\
\text { CI-AKI }(n=10)\end{array}$ & $\begin{array}{l}\text { C } \\
\text { Without CI-AKI }(n=88)\end{array}$ & $p$ value (B vs. C) \\
\hline \multicolumn{5}{|l|}{ Baseline characteristics } \\
\hline Age-years & $61(39-82)$ & $66(57-76)$ & $61(39-82)$ & 0.13 \\
\hline Male gender-no. $(\%)$ & $77(78.6)$ & $9(90.0)$ & $68(77.3)$ & 0.36 \\
\hline Smoking-no. $(\%)$ & $20(20.4)$ & $2(20.0)$ & $18(20.5)$ & 0.97 \\
\hline Paroxysmal atrial fibrillation-no. (\%) & $14(14.3)$ & $3(30.0)$ & $11(12.5)$ & 0.14 \\
\hline Arterial hypertension-no. $(\%)$ & $62(63.3)$ & $7(70.0)$ & $55(62.5)$ & 0.65 \\
\hline Diabetes mellitus - no. $(\%)$ & $34(34.7)$ & $4(40.0)$ & $30(34.1)$ & 0.71 \\
\hline Previous myocardial infarction-no. $(\%)$ & $52(53.1)$ & $5(50.0)$ & $47(53.4)$ & 0.84 \\
\hline Prior $\mathrm{CABG}$ - no. $(\%)$ & $12(12.2)$ & $3(30.0)$ & $9(10.2)$ & 0.07 \\
\hline Previous PCI-no. (\%) & $37(37.8)$ & $4(40.0)$ & $33(37.5)$ & 0.88 \\
\hline Ischaemic cardiomyopathy-no. $(\%)$ & $60(61.2)$ & $6(60.0)$ & $54(61.4)$ & 0.93 \\
\hline NYHA class on admission & $3(3-4)$ & $3(3-4)$ & $3(3-4)$ & 0.37 \\
\hline Left ventricle ejection fraction- $\%$ & $24(12-34)$ & $23(17-34)$ & $24.0(12-34)$ & 0.25 \\
\hline LVESV—mL & $192(94-481)$ & $198(157-321)$ & $189(94-481)$ & 0.39 \\
\hline $\mathrm{LVEDV}-\mathrm{mL}$ & $260(120-586)$ & $272(199-393)$ & $255(120-586)$ & 0.43 \\
\hline Mitral EROA $-\mathrm{mm}^{2}$ & $0(0-48)$ & $0(0-36)$ & $0(0-48)$ & 0.93 \\
\hline Right ventricle diameter $-\mathrm{mm}$ & $31(16-42)$ & $29(24-42)$ & $31(16-42)$ & 0.87 \\
\hline $\mathrm{RVSP}-\mathrm{mmHg}$ & $39(11-73)$ & $38(13-69)$ & $39(11-73)$ & 0.94 \\
\hline QRS complex width—ms & $168(120-220)$ & $174(135-206)$ & $168(120-220)$ & 0.73 \\
\hline $6 \mathrm{MWD}-\mathrm{m}$ & $346(126-488)$ & $316(195-414)$ & $346(126-488)$ & 0.26 \\
\hline Peak oxygen consumption $-\mathrm{mL} / \mathrm{kg} / \mathrm{min}$ & $13.1(7.1-21.0)$ & $13.7(8.5-15.5)$ & $12.9(7.1-21.0)$ & 0.94 \\
\hline NT-proBNP on admission-pg/mL & $1,627(189-28,775)$ & $2,581(1,077-25,890)$ & $1,506(189-28,775)$ & 0.09 \\
\hline C-reactive protein $-\mathrm{mg} / \mathrm{L}$ & $2.3(0.3-105.2)$ & $3.1(0.3-28.6)$ & $2.3(0.4-105.2)$ & 0.96 \\
\hline Complete revascularization-no. $(\%)$ & $68(69.4)$ & $7(70.0)$ & $61(69.3)$ & 0.96 \\
\hline Triple Site CRT—no. (\%) & $48(49.0)$ & $5(50.0)$ & $43(48.9)$ & 0.95 \\
\hline \multicolumn{5}{|l|}{ Renal function parameters } \\
\hline Creatinine on admission $-\mu \mathrm{mol} / \mathrm{L}$ & $94(48-285)$ & $104(86-180)$ & $92(48-285)$ & 0.027 \\
\hline GFR on admission $-\mathrm{mL} / \mathrm{min} / 1.73 \mathrm{~m}^{2}$ & $72.7(20.2-135.7)$ & $65.6(26.2-84.5)$ & $73.8(20.2-135.7)$ & 0.08 \\
\hline Creatinine at discharge $-\mu \mathrm{mol} / \mathrm{L}$ & $138(104-263)$ & $138(104-263)$ & $85(48-242)$ & $<0.001$ \\
\hline GFR at discharge $-\mathrm{mL} / \mathrm{min} / 1.73 \mathrm{~m}^{2}$ & $74.6(16.9-127.7)$ & $46.9(16.9-67.9)$ & $80.7(24.4-127.7)$ & $<0.001$ \\
\hline Creatinine after 6 months $-\mu \mathrm{mol} / \mathrm{L}$ & $117(71-243)$ & $117(71-243)$ & $92(52-198)$ & 0.031 \\
\hline GFR after 6 months $-\mathrm{mL} / \mathrm{min} / 1.73 \mathrm{~m}^{2}$ & $73.4(18.5-150.5)$ & $56.8(18.5-105.4)$ & $75.1(24.7-150.5)$ & 0.059 \\
\hline \multicolumn{5}{|l|}{ Procedural details } \\
\hline Procedure duration: skin to skin $-\min$ & $110(55-225)$ & $115(75-165)$ & $110(55-225)$ & 0.79 \\
\hline Fluoroscopy time $-\min$ & $20.9(4.5-112)$ & $19.7(10.3-39)$ & $20.9(4.5-112)$ & 0.98 \\
\hline Contrast media volume $-\mathrm{mL}$ & $40(0-155)$ & $37(20-125)$ & $40(0-155)$ & 0.79 \\
\hline Use of additional technique $^{\mathrm{a}}$ & $24(24.5)$ & $3(30.0)$ & $21(23.9)$ & 0.67 \\
\hline Intraoperative decompensation ${ }^{\mathrm{b}}$ —no. $(\%)$ & $7(7.1)$ & $0(0.0)$ & $7(7.9)$ & 0.35 \\
\hline Acute reoperation ${ }^{\mathrm{c}} \longrightarrow$ no. $(\%)$ & $4(4.1)$ & $0(0.0)$ & $4(4.5)$ & 0.49 \\
\hline Optimal LV lead position—no. (\%) & $75(76.5)$ & $10(100.0)$ & $65(73.9)$ & 0.068 \\
\hline In-hospital-stay — days & $4(2-19)$ & $3(2-6)$ & $4(2-19)$ & 0.11 \\
\hline \multicolumn{5}{|l|}{ Medication and pacing burden } \\
\hline Beta-adrenergic blocker-no. $(\%)$ & $97(99.0)$ & $10(100)$ & $87(98.9)$ & 0.74 \\
\hline ACE-inhibitor/ARB — no. (\%) & $97(99.0)$ & $10(100)$ & $87(98.9)$ & 0.74 \\
\hline Spironolactone/eplerenon-no. $(\%)$ & $94(95.9)$ & $9(90.0)$ & $85(96.6)$ & 0.32 \\
\hline Loop diuretics-no. $(\%)$ & $91(92.9)$ & $9(90.0)$ & $82(93.2)$ & 0.71 \\
\hline Digoxin-no. $(\%)$ & $10(10.2)$ & $3(30.0)$ & $7(8.0)$ & 0.032 \\
\hline Statin-no. $(\%)$ & $78(79.6)$ & $10(100)$ & $68(77.3)$ & 0.095 \\
\hline
\end{tabular}


Table 1 (continued)

\begin{tabular}{|c|c|c|c|c|}
\hline & $\begin{array}{l}\text { A } \\
\text { Overall population }(n=98)\end{array}$ & $\begin{array}{l}\text { B } \\
\text { CI-AKI }(n=10)\end{array}$ & $\begin{array}{l}\mathrm{C} \\
\text { Without CI-AKI }(n=88)\end{array}$ & $p$ value (B vs. C) \\
\hline Amiodarone - no. $(\%)$ & $6(6.1)$ & $1(10.0)$ & $5(5.7)$ & 0.59 \\
\hline Percentage of CRT pacing_ $\%$ & $99.7(66.0-100)$ & $99.6(82.8-100)$ & $99.7(66.0-100)$ & 0.58 \\
\hline \multicolumn{5}{|l|}{ Outcomes } \\
\hline Responders to CRT-no. $(\%)$ & $86(87.7)$ & $10(100)$ & $76(86.4)$ & 0.21 \\
\hline 1-year hospitalization for $\mathrm{HF}-$ no. $(\%)$ & $19(19.4)$ & $4(40.0)$ & $15(17.0)$ & 0.083 \\
\hline 1-year MACE—no. $(\%)$ & $21(21.4)$ & $4(40.0)$ & $17(19.3)$ & 0.13 \\
\hline 1-year mortality-no. $(\%)$ & $4(4.1)$ & $1(10.0)$ & $3(3.4)$ & 0.32 \\
\hline Remote hospitalization for $\mathrm{HF}-$ no. $(\%)$ & $33(33.7)$ & $4(40.0)$ & $29(33.0)$ & 0.66 \\
\hline Remote MACE—no. (\%) & $34(34.7)$ & $6(60.0)$ & $28(31.8)$ & 0.079 \\
\hline Remote mortality-no. (\%) & $20(20.4)$ & $5(50.0)$ & $15(17.0)$ & 0.016 \\
\hline Cardiac mortality—no. (\%) & $14(14.3)$ & $4(40.0)$ & $10(11.4)$ & 0.016 \\
\hline Sudden deaths-no. $(\%)$ & $4(4.1)$ & $2(20.0)$ & $2(2.3)$ & 0.007 \\
\hline
\end{tabular}

$A C E$ angiotensin-converting enzyme, $A R B$ angiotensin receptor blocker, $C A B G$ coronary artery by-pass grafting, $C I-A K I$ contrast-induced acute kidney injury, $C R T$ cardiac resynchronization therapy, $E R O A$ effective regurgitant orifice area, $H F$ heart failure, $G F R$ glomerular filtration rate, $L V$ left ventricle, $L V E D V$ left ventricle end-diastolic volume, $L V E S V$ left ventricle end-systolic volume, MACE major adverse cardiac event, 6 MWD 6-min walking distance, NT-proBNP N-terminal prohormone of brain natriuretic peptide, $P C I$ percutaneous coronary intervention, $R V S P$ right ventricle systolic pressure

Values presented as median with minimum and maximum or percentage of subjects

${ }^{a}$ Angioplasty or stenting within coronary vein (3 patients), use of active fixation lead (21 patients) or use of sub-selective catheters (7 patients)

${ }^{\mathrm{b}}$ Requiring transient intravenous inotropic support

${ }^{\mathrm{c}}$ Within the same hospital stay, due to lead dislocation (three patients) or phrenic nerve stimulation (one patient)

independent from the treatment arm (conventional vs. triplesite CRT).

\section{Discussion}

The main finding of the present study is that contrast-induced acute kidney injury is a frequent complication of CRT-D implantation and has a significant negative influence on long-term survival. As it has been previously described, contrast-induced acute kidney injury, named also contrast-induced nephropathy

Table 2 Predictors of contrast-induced acute kidney injury after CRT implantation

\begin{tabular}{llc}
\hline Variable & Odds ratio (95\% CI) & $p$ value \\
\hline $\begin{array}{l}\text { Procedure duration: skin to skin } \\
\quad[1 \text { min increase }]\end{array}$ & $1.00(0.97-1.02)$ & 0.80 \\
$\begin{array}{l}\text { Contrast media volume } \\
\quad[1 \mathrm{~mL} \text { increase }]\end{array}$ & $1.00(0.97-1.03)$ & 0.98 \\
$\begin{array}{l}\text { QRS complex width }>\text { median value } \\
\quad[>164 \text { ms }]\end{array}$ & $1.15(0.27-4.90)$ & 0.85 \\
$\begin{array}{l}\text { Left ventricle ejection fraction }> \\
\text { median value }[>24 \%]\end{array}$ & $0.30(0.06-1.59)$ & 0.15 \\
$\begin{array}{l}\text { Serum creatinine level }>\text { median } \\
\text { value }[>94 \mu \mathrm{mol} / \mathrm{L}]\end{array}$ & $6.23(1.19-32.6)$ & 0.03 \\
\hline
\end{tabular}

$C I$ confidence interval, $C R T$ cardiac resynchronization therapy
(CIN), was a serious procedural complication with significant impact on long-term prognosis in subjects undergoing percutaneous cardiovascular procedures [5]. It has been especially well characterized in the population of patients who underwent percutaneous coronary intervention (PCI) or coronary angiography and was associated with a significant worsening of prognosis in this set of subjects [4-6, 13, 14]. Very limited data is however available on the incidence of contrast-induced acute kidney injury in the population of CRT-D recipients, and according to the authors' best knowledge, there is no data on its prognostic value in this set of patients $[8,9]$.

The CI-AKI incidence of $10.2 \%$ in our study is similar to the findings reported by other authors. Actually, there have been so far only two studies in which the frequency of CI-AKI has been assessed $[8,9]$. Cowburn et al. demonstrated $14 \%$ occurrence of contrast-induced nephropathy defined as at least $25 \%$ increase in serum creatinine from the baseline within $48 \mathrm{~h}$ after contrast exposure during CRT implantation [8]. More recently, Tester et al. revealed $8 \%$ incidence of contrast-induced nephropathy after CRT therapy implantation; however, the authors used a different definition of contrast nephropathy, which was a rise in serum creatinine of at least $25 \%$ from the baseline $\geq 48 \mathrm{~h}$ after contrast exposure [9]. High frequency of contrast-induced nephropathy in the study of Cowburn et al. might be the result of both less common use of iso-osmolar, non-ionic contrast media and higher volumes of contrast as well as worse baseline renal function parameters 
A

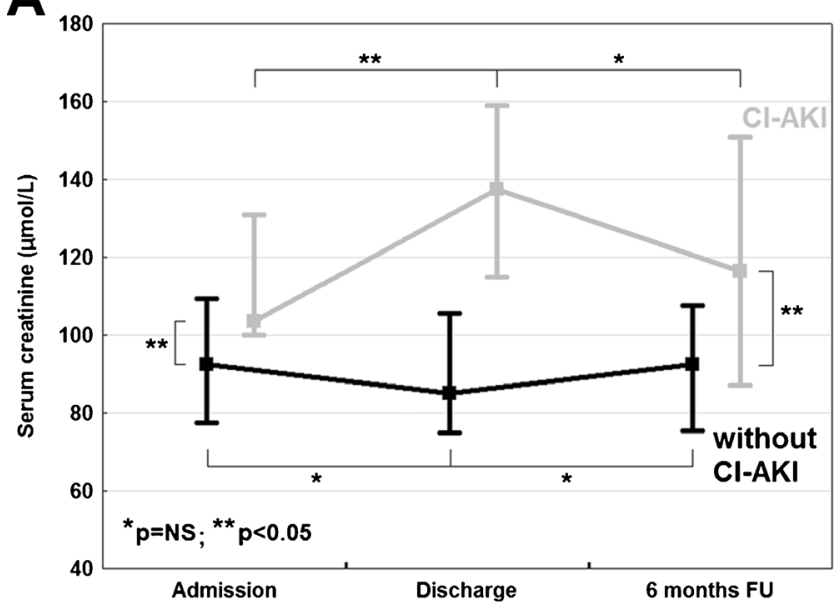

B

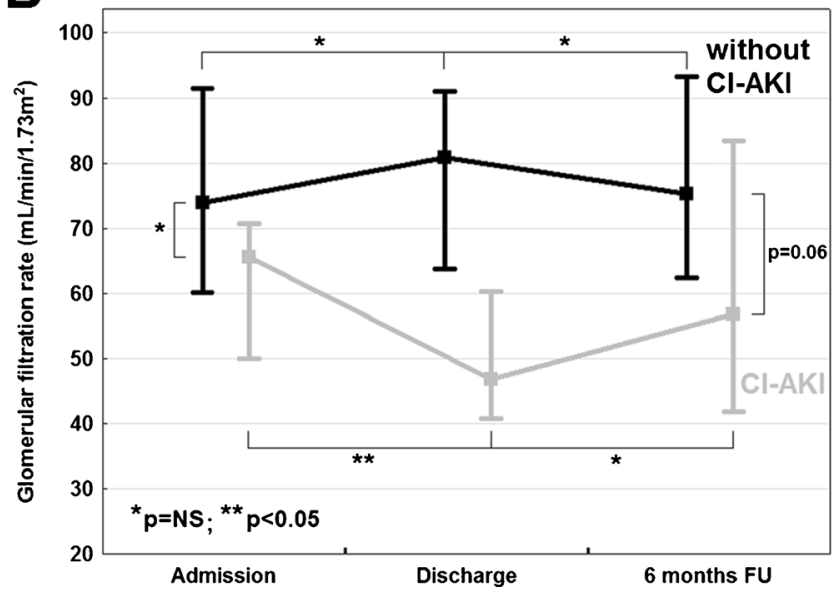

Fig. 1 Changes in renal function estimated with serum creatinine (a) and glomerular filtration rate (b) in CRT-D recipients with respect to CI-AKI occurrence. $C I-A K I=$ contrast-induced acute kidney injury; $C R T-D=$ cardiac resynchronization therapy defibrillator; $F U=$ follow-up; $N S=$ non-significant
Table 3 Independent predictors of death in CRT-D recipients

\begin{tabular}{lll}
\hline Variable & Hazard ratio $(95 \% \mathrm{CI})$ & $p$ value \\
\hline $\begin{array}{l}\text { Peak oxygen consumption } \\
\quad[1 \mathrm{~mL} / \mathrm{kg} / \mathrm{min} \text { increase }]\end{array}$ & $0.81(0.71-0.91)$ & 0.032 \\
QRS complex width [1 ms increase] & $0.97(0.96-0.98)$ & 0.005 \\
Mitral EROA [1 mm ${ }^{2}$ increase] & $1.06(1.05-1.07)$ & $<0.001$ \\
Contrast-induced acute kidney injury & $5.71(5.16-6.26)$ & 0.001 \\
\hline
\end{tabular}

Adjusted for the following: previous percutaneous coronary intervention, NYHA class on admission, NT-proBNP on admission, QRS complex width, mitral EROA, baseline peak oxygen consumption, digoxin at discharge, glomerular filtration rate at discharge, contrast-induced acute kidney injury

$C I$ confidence interval, EROA effective regurgitant orifice area

[8]. For comparison, the incidence of contrast-induced acute kidney injury after PCI ranges from $2.0 \%$ to even $20-30 \%$ with respect to co-morbidities, and mainly depends on the baseline, pre-procedural renal dysfunction, which is one of the most important risk factors of CI-AKI development [4-7, 13, 14]. According to CIN Consensus Working Panel from 2006, contrast-induced nephropathy is responsible for approximately $11 \%$ of hospital-acquired renal failure cases [13]. Thus, these findings coming mainly from registries, where coronary angiograms and PCI were the leading causes of CIN, are similar to the incidence of CI-AKI demonstrated in CRT recipients. Of course, the contrast volumes used during PCI are much higher than during CRT-D implantation. However, on the other hand, patients with chronic heart failure do have very often concomitant chronic kidney disease and thus are at increased risk of CI-AKI development despite relatively small amounts of contrast medium used during the procedure. Twofold increase of the incidence of CI-AKI in patients with GFR
Fig. 2 Kaplan-Meier curves for cumulative survival in CRT-D recipients with respect to $\mathrm{CI}-\mathrm{AKI}$ occurrence. $C I-A K I=$ contrastinduced acute kidney injury; $C R T-D=$ cardiac resynchronization therapy defibrillator

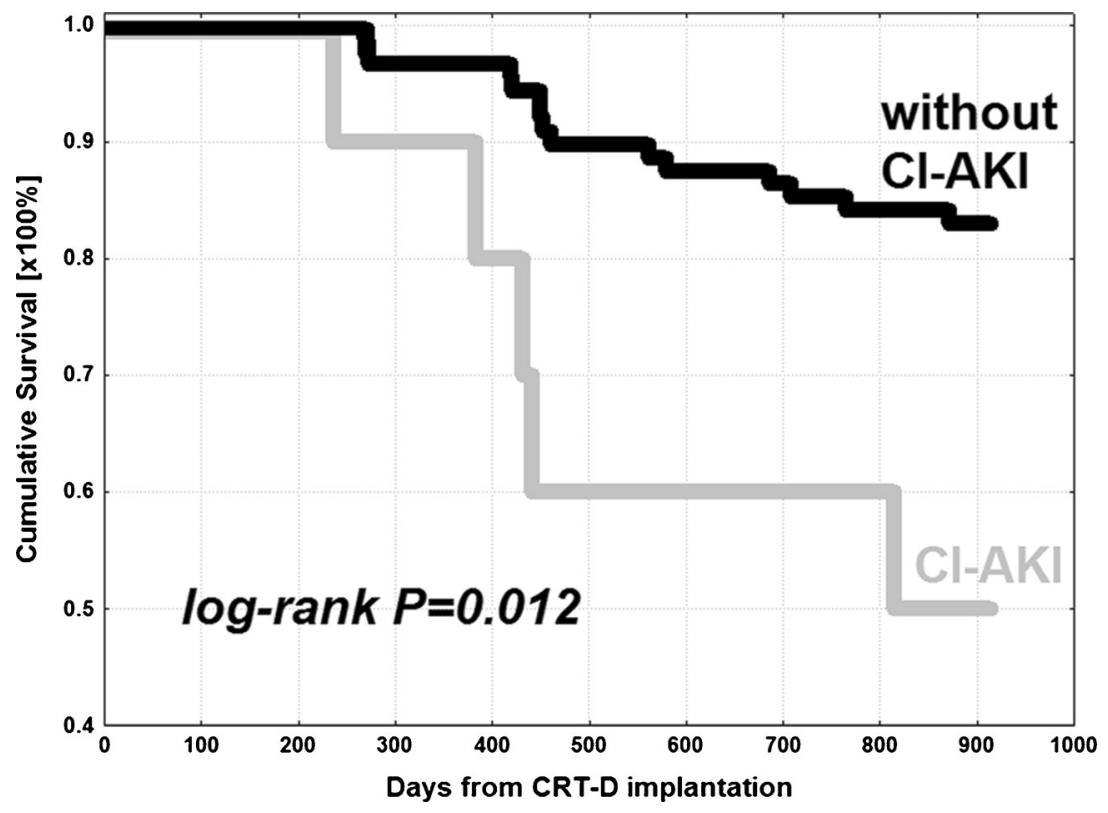


$<60$ on admission, demonstrated in the present study, is consistent with previous observations on increasing risk of contrast-induced nephropathy with declining baseline renal function in the population of PCI patients [15]. It has been stated that one of the most important risk factors of CI-AKI is preexisting chronic kidney disease $[5,7,15]$.

Despite very similar baseline clinical characteristics of CRT-D patients, those with CI-AKI had significantly worse long-term survival than subjects without CI-AKI. Moreover, taking into account cardiac mortality, the significant difference was also observed. The aforementioned two studies on contrast-induced nephropathy after CRT presented data on the incidence of CI-AKI, however without the outcome analysis $[8,9]$. Tester et al. mentioned only that the authors observed a trend towards higher long-term mortality associated with development of CIN [9]. To the authors' best knowledge, there is currently no other data in the literature on the outcome of CRT patients with CI-AKI. It is also worth noting that after 1 year the difference in mortality, though statistically nonsignificant, was considerably high (10 vs. $3.4 \%)$ with its further progression within next months. A very high 30 month mortality in CI-AKI group, almost threefold higher than in patients without CI-AKI, suggests that a very detailed clinical evaluation regarding renal function should be considered in this set of patients, both before and after CRT implantation, with implementation of all clinically proven preventive strategies against CI-AKI. Very high mortality rates in CRT-D recipients, who developed CI-AKI, might be the result of an interaction of many clinical and biological factors such as follows: predisposition to fluid overload leading to higher rates of hospitalization due to heart failure, proneness to electrolyte disturbances that may lead to arrhythmic events and sudden deaths, natural course of renal failure with its all biological consequences like abnormal vascular biology and endothelial dysfunction, hyperactivation of the renin-angiotensin system, anemia, changes in lipids, and disturbances in coagulation.

Similarly to our findings, high mortality rates in the group of CI-AKI patients were also observed by Lin et al. in CRT recipients with GFR $\leq 60$ during 3 years of follow-up [16]. Some other studies have also demonstrated that renal dysfunction is an important predictor of reduced both survival and left ventricle function improvement following CRT [17-21]. The potential confirmation of this phenomenon might be sustained compromised renal function in CI-AKI group after 6 months of followup. Moreover, the fact that serum creatinine and median GFR did not change significantly within 6 months in particular study groups supports the hypothesis that CRT-D may have a limited impact on delaying or preventing deterioration of renal function.

We also observed that contrast exposure is a trigger for some patients with asymptomatic renal failure. Thus, CI-AKI could be a marker of patients with predisposition to overt renal failure, similarly to prediabetic states, which predispose to overt diabetes mellitus.
Another important and not reported previously finding of the present study is that CI-AKI is the strongest independent risk factor of any-cause death in CRT-D recipients, regardless of the GFR value at discharge. Other independent risk factors such as mitral regurgitation, QRS complex width and peak oxygen consumption are well-established risk predictors demonstrated in previously published studies [1, 2, 22-28]. The phenomenon of risk reduction with increasing QRS duration revealed in our study is in line with preceding reports and is explained by a high probability of substantial benefit from CRT therapy observed in CRT recipients with wider baseline QRS complex [22, 23]. The results of multivariate regression analysis with adjustment to GFR at discharge and digoxin usagethe only two differences between study groups, confirmed the finding that CI-AKI had significant impact on long-term prognosis in CRT-D recipients independent of other confounders. This observation is additionally supported by very similar baseline and clinical characteristics of both study groups.

\subsection{Study limitations}

One of the potential limitations of the study is relatively small study population, which could have potentially biased the results. On the other hand, this single-centre randomized study encompassed consecutive heart failure patients, who were treated similarly both invasively (CRT-D implantation) and medically, and this fact makes the study population very homogenous and strengthens the results. The sub-analysis of data from the TRUST CRT trial, which was not primarily designed for the evaluation of CI-AKI in patients undergoing cardiac resynchronization therapy, may also bias the results. Another limitation of the study is lack of complete data from postmortem CRT-D interrogation. Therefore, not all causes of deaths were clear, especially the sudden ones. These data would be helpful in explaining the potential reasons for different long-term prognosis and very high mortality rates in patients with CI-AKI.

In conclusion, CI-AKI is a serious and frequent procedural complication of CRT-D implantation with a significant negative influence on long-term survival independent of other risk factors. The results of the present study should be confirmed in the large cohort studies but they suggest that detailed clinical evaluation regarding renal function should be considered in CRT recipients, both before and after device implantation, with implementation of all clinically proven nephroprotective strategies.

Acknowledgments The trial was registered at ClinicalTrials.gov NCT00814840.

Conflict of interest Dr. R. Lenarczyk do lectures and consultancy for Medtronic and Biotronik; Prof. B. Sredniawa for Boehringer-Ingelheim, Bristol Myers-Squibb, Medtronic, Pfizer, Servier, MSD, Berlin-Chemie, Merck; and Prof. Z. Kalarus for St. Jude Medical, Pfizer, BoehringerIngelheim, Eli Lilly. 
Open AccessThis article is distributed under the terms of the Creative Commons Attribution License which permits any use, distribution, and reproduction in any medium, provided the original author(s) and the source are credited.

\section{References}

1. Brignole, M., Auricchio, A., Baron-Esquivias, G., Bordachar, P., Boriani, G., Breithardt, O.A. et al. (2013). 2013 ESC Guidelines on cardiac pacing and cardiac resynchronization therapy: Addenda. European Heart Journal.

2. Vardas, P. E., Auricchio, A., Blanc, J. J., Daubert, J. C., Drexler, H., Ector, H., et al. (2007). Guidelines for cardiac pacing and cardiac resynchronization therapy: the Task Force for Cardiac Pacing and Cardiac Resynchronization Therapy of the European Society of Cardiology. Developed in collaboration with the European Heart Rhythm Association. European Heart Journal, 28, 2256-2295.

3. Thomsen, H. S., \& Morcos, S. K. (2003). Contrast media and the kidney: European Society of Urogenital Radiology (ESUR) guidelines. British Journal of Radiology, 76, 513-518.

4. Kowalczyk, J., Lenarczyk, R., Kowalski, O., Sredniawa, B., Musialik-Lydka, A., Gasior, M., et al. (2007). Different types of renal dysfunction in patients with acute myocardial infarction treated with percutaneous coronary intervention. Journal of Interventional Cardiology, 20, 143-152.

5. KDIGO AKI Work Group. (2012). KDIGO clinical practice guideline for acute kidney injury. Kidney International. Supplement, 2, 1-138.

6. Lameire, N., Kellum, J. A., \& for the KDIGO AKI Guideline Work Group. (2013). Contrast-induced acute kidney injury and renal support for acute kidney injury: a KDIGO summary (Part 2). Critical Care, 17, 205.

7. Mehran, R., Aymong, E. D., Nikolsky, E., Lasic, Z., Iakovou, I., Fahy, M., et al. (2004). A simple risk score for prediction of contrastinduced nephropathy after percutaneous coronary intervention: development and initial validation. Journal of the American College of Cardiology, 44, 1393-1399.

8. Cowburn, P. J., Patel, H., Pipes, R. R., \& Parker, J. D. (2005). Contrast nephropathy post cardiac resynchronization therapy: an under-recognized complication with important morbidity. European Journal of Heart Failure, 7, 899-903.

9. Tester, G. A., Noheria, A., Carrico, H. L., Mears, J. A., Cha, Y. M., Powell, B. D., et al. (2012). Impact of radiocontrast use during left ventricular pacemaker lead implantation for cardiac resynchronization therapy. Europace, 14, 243-248.

10. Lenarczyk, R., Kowalski, O., Średniawa, B., Pruszkowska-Skrzep, P., Pluta, S., Sokal, A., et al. (2009). Triple-Site Versus Standard Cardiac Resynchronization Therapy Study (TRUST CRT): clinical rationale, design and implementation. Journal of Cardiovascular Electrophysiology, 20, 658-662.

11. Lenarczyk, R., Kowalski, O., Sredniawa, B., Pruszkowska-Skrzep, P., Mazurek, M., Jędrzejczyk- Patej, E., et al. (2012). Implantation feasibility, procedure-related adverse events and lead performance during 1year follow-up in patients undergoing Triple-Site Cardiac Resynchronization Therapy: a substudy of TRUST CRT Randomized Trial. Journal of Cardiovascular Electrophysiology, 23, 1228-1236.

12. National Kidney Foundation. (2002). K/DOQI clinical practice guidelines for chronic kidney disease: evaluation, classification, and stratification. American Journal of Kidney Diseases, 39, S1-S237.

13. McCullough, P. A., Adam, A., Becker, C. R., Davidson, C., Lameire, N., Stacul, F., et al. (2006). Epidemiology and prognostic implications of contrast-induced nephropathy. American Journal of Cardiology, 98, 5K-13K.
14. Marenzi, G., Lauri, G., Assanelli, E., Campodonico, J., De Metrio, M., Marana, I., et al. (2004). Contrast-induced nephropathy in patients undergoing primary angioplasty for acute myocardial infarction. Journal of the American College of Cardiology, 44, 1780-1785.

15. McCullough, P. A., Adam, A., Becker, C. R., Davidson, C., Lameire, N., Stacul, F., et al. (2006). Risk prediction of contrast-induced nephropathy. American Journal of Cardiology, 98, 27K-36K.

16. Lin, G., Gersh, B. J., Greene, E. L., Redfield, M. M., Hayes, D. L., \& Brady, P. A. (2011). Renal function and mortality following cardiac resynchronization therapy. European Heart Journal, 32, 184-190.

17. Kreuz, J., Horlbeck, F., Linhart, M., Mellert, F., Fimmers, R., Schrickel, J., et al. (2012). Independent predictors of mortality in patients with advanced heart failure treated by cardiac resynchronization therapy. Europace, 14, 1596-1601.

18. Verbrugge, F. H., Dupont, M., Rivero-Ayerza, M., de Vusser, P., Van Herendael, H., Vercammen, J., et al. (2012). Comorbidity significantly affects clinical outcome after cardiac resynchronization therapy regardless of ventricular remodeling. Journal of Cardiac Failure, 18, 845-853.

19. Hosoda, J., Ishikawa, T., Matsushita, K., Matsumoto, K., Kimura, Y., Miyamoto, M., et al. (2012). Impact of renal insufficiency on longterm clinical outcome in patients with heart failure treated by cardiac resynchronization therapy. Journal of Cardiology, 60, 301-305.

20. Van Bommel, R. J., Mollema, S. A., Borleffs, C. J., Bertini, M., Ypenburg, C., Marsan, N. A., et al. (2011). Impaired renal function is associated with echocardiographic nonresponse and poor prognosis after cardiac resynchronization therapy. Journal of the American College of Cardiology, 57, 549-555.

21. Mathew, J., Katz, R., St John Sutton, M., Dixit, S., Gerstenfeld, E. P., Ghio, S., et al. (2012). Chronic kidney disease and cardiac remodelling in patients with mild heart failure: results from the REsynchronization reVErses Remodeling in Systolic Left vEntricular Dysfunction (REVERSE) study. European Journal of Heart Failure, 14, 1420-1428.

22. Cleland, J. G., Abraham, W. T., Linde, C., Gold, M. R., Young, J. B., Claude Daubert, J., et al. (2013). An individual patient meta-analysis of five randomized trials assessing the effects of cardiac resynchronization therapy on morbidity and mortality in patients with symptomatic heart failure. European Heart Journal, 34, 3547-3556.

23. Bilchick, K. C., Kamath, S., DiMarco, J. P., \& Stukenborg, G. J. (2010). Bundle-branch block morphology and other predictors of outcome after cardiac resynchronization therapy in Medicare patients. Circulation, 122, 2022-2030.

24. Onishi, T., Onishi, T., Marek, J. J., Ahmed, M., Haberman, S. C., Oyenuga, O., et al. (2013). Mechanistic features associated with improvement in mitral regurgitation after cardiac resynchronization therapy and their relation to long-term patient outcome. Circulation. Heart Failure, 6, 685-693.

25. Di Biase, L., Auricchio, A., Mohanty, P., Bai, R., Kautzner, J., Pieragnoli, P., et al. (2011). Impact of cardiac resynchronization therapy on the severity of mitral regurgitation. Europace, 13, 829-838.

26. Cabrera-Bueno, F., Molina-Mora, M. J., Alzueta, J., PenaHernandez, J., Jimenez-Navarro, M., Fernandez-Pastor, J., et al. (2010). Persistence of secondary mitral regurgitation and response to cardiac resynchronization therapy. European Journal of Echocardiography, 11, 131-137.

27. Richardson, M., Freemantle, N., Calvert, M. J., Cleland, J. G., Tavazzi, L., \& CARE-HF Study Steering Committee and Investigators. (2007). Predictors and treatment response with cardiac resynchronization therapy in patients with heart failure characterized by dyssynchrony: a pre-defined analysis from the CARE-HF trial. European Heart Journal, 28, 1827-1834.

28. Jaussaud, J., Blanc, P., Derval, N., Bordachar, P., Courregelongue, M., Roudaut, R., et al. (2010). Ventilatory response and peak circulatory power: new functional markers of response after cardiac resynchronization therapy. Archives of Cardiovascular Diseases, 103, 184-191. 